\title{
Medios de información, impacto emocional y recomendaciones sanitarias en migrantes venezolanas durante el COVID-19
}

\section{Media, emotional impact and health recommendations on Venezuelan migrant women during the COVID-19}

\author{
Miguel Angulo-Giraldo a* (iD https://orcid.org/0000-0002-5350-9228 \\ Luis Guanipa-Ramírez a (i) https://orcid.org/0000-0002-8934-885X \\ Jose Albites-Sanabria ${ }^{a}$ (i) https://orcid.org/0000-0001-7688-6221
}

Recibido el 15 de octubre de 2020. Aceptado el 16 de agosto de 2021 Publicado el 24 de agosto de 2021.

${ }^{*}$ Autor para correspondencia: Miguel Angulo-Giraldo. Correo electrónico: mangulogi@cientifica.edu.pe

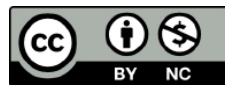

Esta obra está protegida bajo una Licencia Creative Commons Atribución-NoComercial 4.0 Internacional.

\begin{abstract}
${ }^{a}$ Universidad Científica del Sur, Grupo de Investigación MediaLab Científica: Iniciativa de Alfabetización Transmedia, Lima, Perú, correo electrónico: mangulogi@cientifica.edu.pe; lguanipa@cientifica.edu.pe; jalbites@cientifica.edu.pe
\end{abstract}

\section{Resumen}

El presente estudio tiene como objetivo identificar el impacto generado por los medios de información en las mujeres migrantes venezolanas residentes en Lima, Perú, en el contexto del COVID-19, y saber cómo se relaciona con el estado emocional de ellas, así como con el cumplimiento de las recomendaciones sanitarias. Este estudio correlacional retrospectivo recolectó información de 385 de estas mujeres migrantes a través de una encuesta virtual realizada durante las primeras semanas (19 de marzo-30 de abril de 2020) de las medidas de aislamiento social obligatorio tomadas por el gobierno del Perú. Los principales resultados muestran que existe una asociación entre los efectos de los medios y el impacto emocional; ninguno de los medios de comunicación analizados brindó información tranquilizadora, sino inquietante. Este estudio concluye que los medios de comunicación agudizaron los sentimientos de preocupación, miedo, temor y enojo de las mujeres venezolanas migrantes.

Palabras clave: Medios de información, estado emocional, recomendaciones sanitarias, migración, Venezuela.

\section{Abstract}

The objective of this study is to identify the impact generated by the media on Venezuelan migrant women who live in Lima, Peru, in the context of the COVID-19, and to know how it relates to the emotional state, and to the compliance with health recommendations. This retrospective correlational study collected information from 385 of these migrant women, through a virtual survey conducted during

CÓMO CITAR: Angulo-Giraldo, M., Guanipa-Ramírez, L. \& Albites-Sanabria, J. (2021). Medios de información, impacto emocional y recomendaciones sanitarias en migrantes venezolanas durante el COVID-19 [Media, emotional impact and health recommendations on Venezuelan migrant women during the COVID-19]. Estudios Fronterizos, 22, e075. https://doi.org/10.21670/ref.2112075 
the first weeks (March 19-April 30, 2020) of the mandatory social isolation measures taken by the Peruvian government. The main results show that there is an association between media effects and emotional impact; none of the analyzed media provided reassuring information, but disturbing information. This study concludes that the media sharpen the feelings of concern, fear, terror and anger of Venezuelan migrant women.

Keywords: Media, emotional state, health recommendations, migration, Venezuela.

\section{Introducción}

COVID-19 es la enfermedad infecciosa más recientemente descubierta de la familia de los coronavirus que mantiene en alerta a la salud pública global. Inició en Wuhan (China) en diciembre de 2019 y para marzo de 2020 se había propagado a los cinco continentes (World Health Organization [who], 2020). En América Latina, el Perú es el cuarto país de América Latina con mayor cantidad de casos de coronavirus (835 662), detrás de Brasil (5 028 444), Colombia (886 179) y Argentina (856 369) (Ministerio de Salud, 2020; Infomed-Centro Nacional de Información en Ciencias Médicas, 2020). ${ }^{1}$ Las reacciones gubernamentales comenzaron con la declaratoria de emergencia, las medidas de aislamiento social obligatorio, además del cierre de fronteras y la paralización de industrias no esenciales.

En ese contexto, el Perú ha adoptado medidas de apoyo económico para poder contribuir a que las poblaciones vulnerables (trabajadores informales, trabajadores independientes, entre otros) puedan sobrellevar la emergencia (Aguirre, 2020; Fowks, 2020). Como parte de esas medidas, el 23 de abril se anunció un bono universal para todos los peruanos que figuran en el Registro Nacional de Identificación y Estado Civil (Reniec) y que no cuenten con un trabajo formal y no hayan sido beneficiados por otros bonos, lo cual se estipuló en el Decreto de Urgencia 052-2020. Si bien las medidas estatales han sido relevantes para apoyar a la población en el territorio peruano, los migrantes que ahí residen no han sido objeto de estas.

Según cifras del Alto Comisionado de las Naciones Unidas para los Refugiados (ACNUR) y la Organización Internacional para las Migraciones (OIM, 2020), el total de migrantes, refugiados y solicitantes de asilo venezolano que dejaron su país es de $5202270 .{ }^{2}$ El Perú es el segundo lugar con mayor cantidad de refugiados y migrantes venezolanos a nivel mundial: residen cerca de 862000 venezolanos y más de 482500 han solicitado el reconocimiento de ser refugiados (ACNUR, 2020).

Las cifras del Instituto Nacional de Estadística e Informática (INEI, 2018, pp. 22, 53) indican que $80 \%$ de los venezolanos residentes en Perú están en Lima, principalmente en distritos del norte de Lima y el distrito más grande del Perú, San Juan de Lurigancho. Además, $70 \%$ de los migrantes venezolanos en Perú tienen entre 18 y 45 años de edad, $60 \%$ de este grupo arribó al Perú con al menos un familiar, y $75.3 \%$ vive principalmente con familiares.

Sus condiciones sociodemográficas permiten visualizar su precariedad: de $11 \%$ que padece problemas de salud crónica, $77.9 \%$ no recibe tratamiento, además,

\footnotetext{
${ }^{1}$ Cifras al 13 de octubre de 2020

${ }^{2}$ Cifra al 5 de junio de 2020.
} 
91.5\% no cuenta con un seguro de salud. La situación laboral es igualmente precaria, si bien $78.3 \%$ es dependiente asalariado, $88.5 \%$ de estos no tiene contrato laboral (INEI, 2018, pp. 65, 103).

Es importante notar que la variable de género marca una diferencia entre los migrantes venezolanos: si bien las mujeres representan $47.7 \%$ del total, más de $50 \%$ de ellas trabaja más de 60 horas semanales y su promedio de pago mensual es de S/1 026 (US\$ 293), el cual es inferior en S/157 (US\$ 45) al de los hombres que trabajan en la misma actividad laboral que ellas. Igualmente, los rubros principales en los que laboran son cocineras y ayudantes de cocina $(19.1 \%)$; meseras o camareras $(16.9 \%)$ y limpiadoras o asistentes domésticas (10.8\%) (INEI, 2018, p. 108).

Además, en el escenario social, la repercusión de la migración venezolana ha evidenciado los estereotipos y prejuicios que posee la sociedad peruana frente a los migrantes. En ese sentido, $70 \%$ de la población peruana considera que estos migrantes tienen un impacto negativo en el país (Maeda Jerí, 2021), lo cual grafica un problema presente en América Latina en el cual el "extranjero" es visto como un "otro" que representa una "amenaza a la identidad nacional desde fuera y corroe la nación” (Hopenhayn \& Bello, 2001, p. 10). En este contexto, como señala Maeda Jerí (2021, p. 12), los medios de información han contribuido a construir un "ideario del migrante venezolano como el enemigo y además el responsable de todos los males del país”.

Fleury (2016) señala que las mujeres migrantes, por su condición de género, viven una situación particular: si bien la migración permite el empoderamiento de las mujeres - al acceder a trabajo y educación - lo cual mejora la igualdad de género; también se exacerban sus vulnerabilidades entre las que figuran el abuso, la trata y el trabajo sexual. Entre las características principales que viven las mujeres migrantes destacan: la segregación de género en relación con la fuerza laboral —cuando las mujeres migran, a menudo trabajan en posiciones "femeninas" (sectores de servicio o doméstico), lo cual implica que se les percibe como más "dóciles" y "más baratas"-, esto ocurre tanto con las mujeres de profesiones poco calificadas como con las altamente calificadas; altas tasas de desempleo respecto de los hombres migrantes o mujeres locales; son más susceptibles al abuso y tienen menos capacidad de asociarse entre ellas, lo cual repercute en la creación de redes de apoyo y la toma de conciencia sobre derechos colectivos e individuales; enfrentan abusos, discriminación en sus lugares de trabajo y son más vulnerables a la violencia; y, finalmente, no cuentan con un acceso confiable a la atención médica.

En el caso específico de las mujeres, los medios de información han construido una idea acerca de las venezolanas migrantes que afectan no solo la imagen de ellas, sino la imposibilidad de acceder a un entorno libre de maltratos, discriminación y acoso: "la mujer migrante se encuentra en la otredad cultural, siendo invisibilizada al momento de ver sus necesidades y ser considerada dentro de los procesos de migración, relegando sus capacidades al espacio privado y, en algunos casos, hipersexualizando su figura" (Blouin, 2019, p. 98).

El rechazo hacia las mujeres venezolanas trasciende así la construcción mediática y social para materializarse en acciones específicas. Estas mujeres son rechazadas, en muchos casos, del ámbito laboral, ya sea directa o indirectamente por factores relacionados con su condición de un "otro" migrante, un extranjero (Koechlin \& Eguren, 2018, p. 91). Esto se agudiza cuando se reconoce incluso la exclusión laboral en relación con un imaginario colectivo emocional: "las mujeres venezolanas 
encuentran más difícil conseguir empleo, por los celos de las parejas de contratistas peruanos hombres, por como lucen físicamente” (Velarde Ramirez, 2019, p. 9).

Respecto de los antecedentes de esta investigación, es importante observar el incremento exponencial de investigaciones sobre los impactos del COVID-19 en diversos escenarios. En relación con el estado emocional, en este contexto, la base de datos Dimensions (Scopus, WoS y otros) presenta solo cinco estudios que enfatizan el impacto emocional de coronavirus en la vida de distintos grupos poblacionales: Limcaoco y colaboradores (2020, p. 3) encuestaron a ciudadanos de 25 países con el fin de medir la ansiedad, el miedo y el estrés percibido, dentro de lo que destaca el incremento en los niveles de ansiedad; Vidyadhara y colaboradores (2020, p. 4) y Venigalla y colaboradores (2020, p. 6) trabajan con ciudadanos de la India: el primero investiga a los estudiantes de farmacia del sur de este país, mientras el segundo mide el estado emocional a través de las reacciones en la red social Twitter; Lima y colaboradores (2020) muestran que "During disease outbreaks, community anxiety can rise following the first death, increased media reporting, and an escalating number of new cases" (p. 1); y, finalmente, en un artículo de revisión documental, Brooks y colaboradores (2020, p. 45) observan que las mujeres embarazadas podrían obtener beneficios en el acceso a información en el contexto del COVID-19.

En la misma base de datos no se obtuvieron resultados sobre artículos referentes a los efectos de los medios de información en la población relacionados con la información sobre el coronavirus, mientras que sobre el cumplimiento de las recomendaciones sanitarias se distinguen seis estudios: Marasinghe $(2020$, p. 2) estudia las recomendaciones sobre el uso de mascarilla en población asintomática en la fase inicial de la pandemia; Irvine y colaboradores (2020, p. 442) analizan los casos de los inmigrantes indocumentados detenidos en las instalaciones del Servicio de Inmigración y Control de Aduanas (ICE, por sus siglas en inglés) de Estados Unidos de América y da cuenta de la alta probabilidad $(72 \%)$ de que todos los detenidos se infecten en los siguientes 90 días por las condiciones de hacinamiento; Ammar y colaboradores (2020, p. 3) estudian el efecto de las recomendaciones sanitarias en la actividad física de las personas en cinco continentes y concluye que el confinamiento altera la actividad física y los comportamientos alimenticios en una dirección que compromete la salud; Patel y Jernigan (2020, p. 142) y Jernigan (2020, p. 2) estudian las reacciones del gobierno de Estados Unidos en la fase inicial de la epidemia para observar cómo se aplicaron las recomendaciones sanitarias; finalmente, Khan y colaboradores $(2020$, p. 215) concluyen la necesidad de tomar en cuenta las recomendaciones sanitarias de la Organización Mundial de la Salud (oms) (lavarse las manos, cubrirse la boca al toser, etcétera) con el fin de "minimize the risk of exportation or importation of the disease" (who, 2020, p. 2).

El presente texto se estructura de la siguiente manera, primero presenta el marco teórico de referencia en relación con las variables estudiadas (impacto emocional, efectos de los medios de información y recomendaciones sanitarias), así como la interrelación entre estas; seguidamente muestra el método científico aplicado, para a continuación exponer los hallazgos obtenidos y se finaliza con la discusión y conclusiones de los autores. 


\section{Marco teórico de referencia}

\section{Impacto emocional}

El ser humano como ser biopsicosocial se enfrenta a diversas vicisitudes o adversidades que pueden afectar o alterar su estado psicoemocional, de acuerdo con los estímulos percibidos por las condiciones ambientales, de modo que la interacción sociocultural es fundamental en su desarrollo emocional (Mulsow, 2008, p. 64). Sin embargo, las convulsiones provocadas por el mundo postmoderno, la crisis de los mercados globales, la influencia de las redes sociales, entre otras preocupaciones están generando esferas negativas en el estado emocional del individuo, que provocan desesperación, insensatez, ira incontrolada, violencia, abuso, enfermedad, lo que desemboca en estadios de desequilibrio emocional en el hombre (Goleman \& Cherniss, 2013, p. 216). Estos factores ocupan una mayor relevancia en el contexto de la pandemia de COVID-19, la cual desencadena distintos episodios emocionales.

$\mathrm{Si}$ bien las emociones se definen como un estado complejo caracterizado por una emoción o disturbio que conducirá a una acción; como la respuesta a un evento externo o interno que enfrenta el sujeto; el impacto emocional se concibe como una consecuencia psicosocial, ya que surge principalmente de un conjunto de estímulos contextuales a los que está expuesto el individuo (Serrano \& Ibáñez, 2015, p. 484). En ese sentido, esta investigación pretende medir cómo el contexto del COVID-19 y sus posteriores consecuencias sociales, políticas, laborales y económicas impactaron en la vida de las mujeres migrantes venezolanas residentes en Lima.

Para medir adecuadamente esta variable se toma en cuenta una clasificación de seis categorías: miedo, sorpresa, disgusto, ira, alegría y tristeza (Serrano \& Ibáñez, 2015, pp. 465-466; Ekman \& Friesen, 1978, p. 50). Esta clasificación es también señalada por Ponce de León y colaboradores (2010, p. 10) y tiene una aplicación en un contexto de pandemia, por lo que estas serán las dimensiones consideradas al medir el impacto emocional, es decir, al reconocer el "estado emocional actual frente a la situación de salud experimentada”.

\section{Efecto de los medios de información en la población}

La construcción de la realidad por los medios de información incide no solo en la colocación de un tema dentro de la agenda pública (agenda setting), sino también en la presencia de una perspectiva o enfoque (frame) que interpreta esa realidad en los usuarios. La construcción mediática configura y reconfigura las fronteras de la agenda pública y, en ese sentido, da forma a la realidad, al establecer representaciones sociales e imaginarios colectivos (Gutiérrez San Miguel et al., 2010, p. 283).

No obstante, la construcción de la realidad social también es reapropiada por los ciudadanos en sus espacios de socialización (familia, escuela, redes sociales, etcétera); de manera que, como destaca Reguillo (1998, p. 26), prevalecen "nudos de tensión desde los que se negocian o se oponen en conflicto distintos significados sociales, en y sobre la ciudad y el mundo". En el contexto presente, estos espacios de socialización se transforman gracias a las plataformas digitales, de manera que, los dispositivos 
mediáticos digitales "contribuyen a reconfigurar numerosos aspectos de la vida cotidiana y así como de los procesos de subjetivación y socialización contemporáneos" (Lasén Díaz, 2014, p. 7).

Finalmente, en esta construcción social, los sujetos son interpelados no solo por los medios de comunicación masiva sino por un amplio conjunto de emisores informativos, sumado a las prácticas históricas individuales y grupales de los sujetos. Si bien en los últimos años los medios sociales digitales han tenido relevancia en la construcción de la realidad social, principalmente por ser una amplia red de socialización que cada vez tiene una mayor presencia, estas también han contribuido a incrementar el capital social de los usuarios (Pastrana Valls, 2017, p. 31). De esta manera, las redes sociales digitales y las no digitales en las cuales los ciudadanos participan constituyen un imaginario socialmente compartido en constante reconfiguración (Cogo, 2014, p. 4).

A partir de lo anterior, la idea de efecto de los medios de información hace referencia a que estos ejercen un "impact on the individual's life in both a positive and negative way, influencing all age groups in one direction” (Adewunmi et al., 2021, p. 165). Así, la información enviada y compartida por una amplia red de información —en la cual los medios de comunicación y líderes de opinión destacan sobre los otros- afecta a los sujetos, sin embargo, esta información tiene un impacto mediado, es decir, es reconstruida y recuperada por los propios sujetos en sus entornos de socialización tanto online como offline (Angulo-Giraldo \& Bolo-Varela, 2021).

\section{Recomendaciones sanitarias durante la pandemia por COVID-19}

Los gobiernos de los países más afectados por la pandemia de COVID-19 han tomado una serie de disposiciones para evitar, en la medida de lo posible, la propagación del virus. Cuando se habla de recomendaciones sanitarias desde y para los ciudadanos comunes hay que pensar en dos planos: uno dirigido a no contraer la enfermedad, en el plano individual y otro a no propagar el virus, en el plano social. Como en todas acciones que se planifican, como afirma Guanipa Ramírez (2019, p. 30), es necesario observar a los Estados como macroorganizaciones donde existe un equilibrio entre lo individual (los ciudadanos) y lo colectivo (los gobiernos locales, regionales y nacionales).

En el plano individual, las medidas van a estar enfocadas, principalmente, en un estricto cuidado de la higiene personal y de las áreas del lugar de habitación, así como de los objetos que entren en el mismo. Lo primero, según la Organización Mundial de la Salud (wHo, 2020, pp. 1-2), es importante optimizar el aseo de las manos, para ello se recomienda limpiar las manos con abundante agua y jabón, si se está en casa, y si es necesario salir, usar de forma periódica algún desinfectante a base de alcohol (sobre todo al entrar en contacto con objetos potencialmente infectados). Del mismo modo, se debe asegurar la limpieza de los ambientes de la casa que hayan estado en contacto con elementos provenientes del exterior, así como la desinfección de los productos del mercado. De esta manera se estarán colocando barreras sanitarias en los hogares, lo que reduciría el riesgo de entrada del virus.

Todavía en el plano individual, si la persona tiene que salir de casa, bien sea por motivos laborales o para la compra de bienes de primera necesidad, es necesario que tome ciertas medidas-barrera. En primer lugar, debe cubrirse la nariz y boca usando una mascarilla, esta es una primera línea de defensa para evitar inhalar posibles 
partículas del virus, presentes en el aire, además de no ser agente de contagio, si se es portador asintomático. En segundo lugar, se debe mantener cierta distancia entre las personas con las que se comparte el ambiente y evitar cualquier tipo de contacto físico. Al llegar a casa asegurarse de lavar la ropa que se llevaba puesta durante la salida, así como realizar el lavado de manos que se mencionó antes.

En el plano social, la principal medida recomendada es el aislamiento. Esta acción, según Jefferson y colaboradores (2011, párr. 1), resulta eficaz como barrera de transmisión de cualquier virus. En el caso de la pandemia por COVID-19 ha sido la estrategia implementada por la mayoría de los gobiernos en el mundo. Esta medida debe ser acatada de forma estricta sobre todo por personas de 50 años o más, quienes representan, según el Nuevo Equipo de Epidemiología de Respuesta a Emergencia de Neumonía por Coronavirus (Surveillances, 2020, párr. 7), 81\% de los fallecidos por Coranavirus en China hasta el 11 de febrero. Otro de los grupos poblacionales de alto riesgo son las personas en situaciones de comorbilidad, como enfermedades cardiovasculares, hipertensión arterial o diabetes.

\section{¿Existe relación entre la información de los medios de información, el cumplimiento de las recomendaciones sanitarias y el impacto emocional?}

Las informaciones que aparecen en todos los espacios sociales en los que circulan los ciudadanos impactan en distinta medida en su vida diaria, de esta manera, se reconoce que las representaciones que los medios informativos y los espacios sociales construyen acerca de la realidad tienen un efecto en ellos (Durkin \& Wakefield, 2008, p. 667; Ma \& Stahl, 2017, p. 303; Stanley et al., 2017, p. 648). Este efecto se hace más notorio en contextos de emergencia, tales como epidemias o desastres sociales (Akingbade, 2018, p. 139; Moore \& Friedsam, 1959, p. 135; Rodríguez, 2006, p. 178; Wang, 2006, p. 250; Yang \& Ma, 2020, párr. 1), lo cual amerita una conciencia ética de responsabilidad informativa no solo de los ciudadanos como individuos, sino, sobre todo, de los medios de comunicación, los cuales deberían contribuir a no alarmar a la población, sino a reconocer las maneras de actuar.

Para el caso específico de la población migrante, la importancia de la labor de los medios de comunicación al construir representaciones sociales sobre ellos reside en que "The metaphor of immigrant as pollutant articulated in popular discourse is significant for the ways in which it constructs immigrant, through racial and xenophobic stereotypes, as object, aberrations, and dangers" (Cisneros, 2008, p. 590). En esta línea, Valenzuela-Vergara (2019) - a partir de un caso en Chile- destaca que los medios construyen una visión dual (buenos/malos) sobre los migrantes en la cual un grupo de estos son criminales que deben ser castigados, mientras que los otros carecen de beneficios sociales, por lo que deben ser protegidos y cuidados; entretanto, Kalfeli y colaboradores (2020) —en el caso de Grecia— señalan que existen dos subtramas en la narración mediática sobre los migrantes: una primera de conflicto directo en la cual se estereotipa a los inmigrantes, se incide en la inmigración como un problema social y se polariza el tema; y un segundo enfoque de conflicto indirecto que se caracteriza por "systematically not using migrant voice as a source, by focusing only on negative events around immigration, by not debunking stereotypes [...] consistently leaves critical pieces of information out of its content" (p. 14). 
A partir de lo anterior, el problema principal de la realidad informativa no solo está en el lenguaje empleado, sino en la función de este como una acción social que repercute en la inclusión de los otros (Rosa, 2012). Así, las representaciones inciden en el espacio social, de manera que deshumanizan la agencia y refuerzan los estereotipos comunes. La identidad primaria de los inmigrantes está marcada por su diferencia racial y su estatus de inmigrante ilegal. Sus cuerpos marrones son retratados como sucios y peligrosos debido a su origen étnico³ (Cisneros, 2008, p. 591).

La información promovida desde distintos actores sociales — principalmente los medios de comunicación- afecta los entornos de personas en situaciones de vulnerabilidad, sobre todo afecta la forma en la que los distintos grupos sociales construyen una imagen sobre ellos: "[the] perceptions about people and communities can be incredibly potent and compelling when it comes to prejudices about how they are depicted in the media", de manera que "much of our views and behaviors towards certain classes of people could be based [...] on what we see in the media" (Issaka, 2021, p. 2).

Finalmente, para situarse en un contexto de pandemia, vale distinguir el enfoque de amplificación social y atenuación del riesgo (Kasperson et al., 1988) para comprender que los medios de comunicación provocan un incremento del riesgo percibido frente a una emergencia sanitaria, lo cual tiene como causa el tratamiento que le otorgan los medios al contexto (Kasperson \& Kasperson, 1996, p. 95). La influencia de los medios de comunicación para cumplir recomendaciones sanitarias ha sido demostrada en investigaciones precedentes (Eggener, 1998, p. 1400; Mboera \& Rumisha, 2004, p. 76) e incluso, en el contexto de COVID-19, se conoce que la información de los medios de comunicación ha sido fundamental para el cumplimiento de medidas sanitarias que contribuyen a la no propagación del COVID-19 (La et al., 2020).

De esta forma, los antecedentes demuestran la existencia de relaciones entre las tres variables estudiadas, por lo que esta investigación busca demostrar si en el contexto del COVID-19 existió una relación entre el impacto emocional, los efectos de los medios de comunicación y el cumplimiento de las recomendaciones sanitarias, específicamente por las mujeres venezolanas migrantes residentes en Lima.

\section{Materiales y métodos}

De acuerdo con el paradigma asociativo, el diseño de esta investigación es correlacionalexplicativa, ya que el investigador recaba los datos de la realidad con el fin de encontrar relaciones entre ellos y se intentan explicar las razones que conllevaron a estas actitudes por la población estudiada. Asimismo, este trabajo se identifica como uno retrospectivo y transversal en tanto recoge información sobre alguna característica de interés en la población en un único momento.

En relación con la población, se decidió metodológicamente trabajar únicamente con las mujeres migrantes venezolanas dada su condición mayoritaria en la población

\footnotetext{
${ }^{3}$ Traducción propia de "(...) dehumanize them agency and reinforcing common stereotypes. Inmigrants' primary identity is marked by their racial difference and illegal migrant status. Their brown bodies as portrayed as dirty and dangerous because of their ethnicity" (Cisneros, 2008, p. 591).
} 
peruana $-58 \%$ de los migrantes venezolanos que residen en Lima, Perú, son mujeres, es decir, aproximadamente 483000 personas (Mendoza \& Miranda, 2019, p. 501; Vargas, 2019, párr. 2)—, además de la situación de mayor vulnerabilidad de este grupo para afrontar la crisis del COVID-19, lo cual se agrava por las condiciones propias de la migración (Organización de las Naciones Unidas [ONU], 2019, p. 20; Portal de Datos Mundiales sobre la Migración, 2021; Programa de las Naciones Unidas para el Desarrollo [PNUD], 2020, p. 10; Wenham et al., 2020, p. 846; Women's link worldwide et al., 2020, pp. 6-7). Las razones de la elección de la unidad de análisis no repercutieron en el desarrollo metodológico del estudio.

Así, al considerar como unidad de análisis a las mujeres migrantes se optó por recurrir a dos asociaciones de migrantes venezolanos residentes en Lima: ong Unión Venezolana y Asociación Pasos Firmes. La primera es una asociación sin fines de lucro fundada en 2015 cuyo objetivo es impulsar proyectos de regularización migratoria e integración de los venezolanos en Perú, ubicada en Miraflores (Lima, Perú), pero de alcance nacional. La segunda es una asociación civil humanitaria conformada por 12 personas y fundada el 21 de julio de 2019, cuyo objetivo está centrado en apoyar a las mujeres, niñas, niños y adolescentes migrantes residente en Perú, y su centro de influencia se ubica en Comas (Lima, Perú).

Se tuvo una muestra no probabilística por conveniencia. Dicha muestra tuvo una prevalencia máxima a la exposición de $50 \%$, un margen de error de $5 \%$ y un nivel de confianza de $95 \%$. La muestra de 384 personas debió aumentarse en $10 \%$ por pérdida o rechazo por lo que se obtuvo finalmente un tamaño muestral de 422 (Figura 1).

Figura 1. Diagrama de flujo de selección de universo y muestra

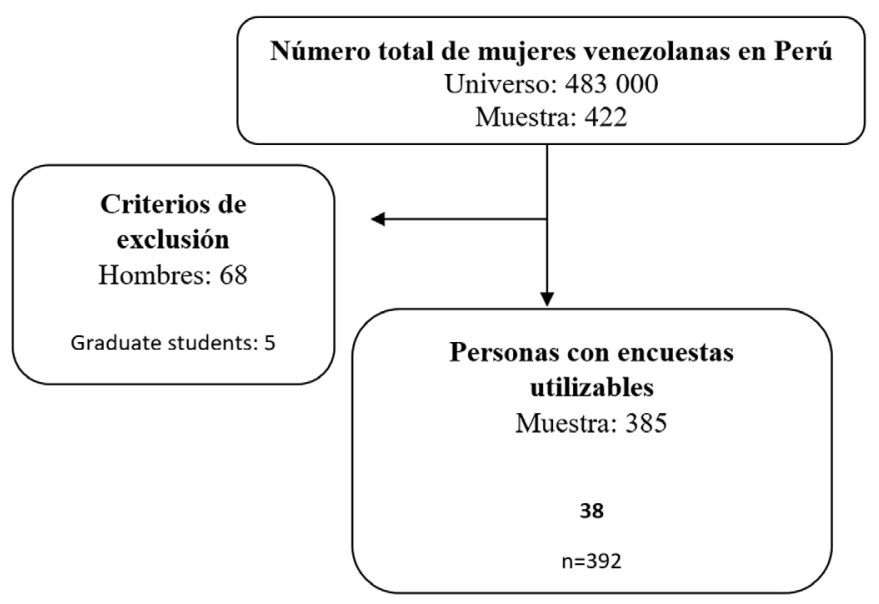

Fuente: Elaboración propia

Se aplicó un cuestionario adaptado del trabajo original de Ponce de León y colaboradores (2010, p. 10) para las tres variables analizadas y modificado para contextualizarlo frente al COVID-19. Inicialmente, se hizo un conjunto de preguntas relacionadas con las características sociodemográficas de la población: formación académica (secundaria incompleta, secundaria completa, universitaria completa, universitaria incompleta, técnica completa, técnica incompleta, posgrado completa, 
posgrado incompleta); condición laboral actual (si cuenta con un contrato de trabajo o no); actividad laboral (respuesta abierta); características de las personas con quienes vive en casa (identificado a partir de las personas con las que vive en casa entre hermanos, hijos, sobrinos, amigos, parejas, abuelos, etcétera) ${ }^{4}$ cantidad de personas con quienes vive (desde ninguna hasta más de 7); tiempo de residencia que tiene en Perú (desde menos de 1 año hasta los 5 años); tipo de documento de identificación que posee (Permiso Temporal de Permanencia [PTP] activo o vencido, carné de extranjería o pasaporte con visa humanitaria, carné de solicitante de refugio o el trámite del mismo, otro documento y no deseo responder ${ }^{5}$ ); y, acceso al sistema de salud durante los últimos tres meses (atención ambulatoria, Seguro Integral de Salud [sis], EsSalud, seguro privado, seguro de Entidad Prestadora de Salud [EPs] u otro).

La variable impacto emocional se evaluó a partir de la confluencia de la calificación de su estado emocional en el momento actual de la situación frente al COVID-19 ("tranquilo", "preocupado", "temeroso", "aterrado", “enojado", "desorientado" y "emocionado"); con la escala de valoración estimativa tipo Likert ("nada", "muy poco", "poco", "regular", "mucho" y "muchísimo").

La variable efectos de los medios masivos de información tomó en cuenta la información distribuida por la prensa, la televisión, la radio, el centro de trabajo, los compañeros o jefes de trabajo, la universidad, las conferencias de prensa y los mensajes a la nación del presidente Martín Vizcarra, los grupos de WhatsApp con familiares y compañeros de trabajo, los grupos de WhatsApp con amigos, las redes sociales (Twitter y Facebook) y los mensajes de texto. Estos se evaluaron con las opciones sugeridas por Ponce de León y colaboradores (2010): "información tranquilizadora", "inquietante" o "ninguna".

Finalmente, para la variable cumplimiento de las recomendaciones sanitarias implementadas por el gobierno peruano se consideraron tres medidas: el aislamiento social; el lavado frecuente de manos con agua y jabón por 20 segundos; y, cubrirse la nariz y la boca con el antebrazo o pañuelo desechable al toser. Las opciones de respuesta se clasificaron en: "siempre", "casi siempre", "a veces", "casi nunca" y "nunca".

La encuesta fue realizada entre el 19 de marzo y el 30 de abril de 2020, en el contexto de emergencia sanitaria y aislamiento social obligatorio declarado en Perú el 16 de marzo de 2020. Las asociaciones con las cuales se trabajó difundieron las encuestas en los grupos de WhatsApp en los que están incluidos todos sus asociados. El mensaje que enviaba el líder de la organización (Asociación Pasos Firmes y Unión Venezolana) pedía el llenado de las encuestas principalmente por las mujeres. De esta manera, se distribuyó de manera virtual a través de las dos instituciones gracias a los grupos que ambas tienen.

Con las encuestas llenas se procedió a hacer las correcciones respectivas en el programa Microsoft Excel. Luego, se procesó la información en el software Python versión 3.8.2. Se consideró que la significancia es $\mathrm{p}<0.05$.

\footnotetext{
${ }^{4}$ Para clasificar las respuestas a esta pregunta, se utilizó la categorización que sugiere Vargas Murga (2014, p. 57): la familia extensa ("más de dos generaciones en el mismo hogar"); la familia nuclear ("padres y sus hijos"); la familia nuclear que tiene parientes próximos que residen con ellos; la familia nuclear que no cuenta con otros parientes próximos; la familia nuclear numerosa; la familia nuclear ampliada ("con parientes o con agregados"); la familia monoparental en la cual solo vive uno de los cónyuges con sus hijos; la familia reconstituida ("formada por dos adultos en la que al menos uno de ellos, trae un hijo habido de una relación anterior"); las personas sin familia (las cuales viven solas); y los equivalentes familiares (personas que conviven en un mismo hogar sin constituir un núcleo familiar tradicional).

${ }^{5}$ La opción de no responder fue incluida ya que las condiciones de vulnerabilidad de los migrantes venezolanos en Perú ameritan que exista temor por parte de esta población a revelar que su condición migratoria es irregular, lo cual implicaría un problema para continuar viviendo en el país.
} 
Debido a la situación de emergencia epidemiológica, los individuos aceptaron participar en el estudio en estricto cumplimiento de las consideraciones éticas y en consideración de la Ley de Protección de Datos Personales (29733) del Perú. Todos los que llenaron la encuesta aceptaron que sus datos fueran utilizados únicamente para esta investigación.

\section{Resultados}

Tabla 1. Características de la población en relación con la formación académica

\begin{tabular}{|l|c|c|}
\hline Formación académica & $\mathbf{N}$ & $\%$ \\
\hline Educación universitaria de pregrado completa & 114 & 29.6 \\
\hline Secundaria completa & 79 & 20.5 \\
\hline Educación técnica completa & 70 & 18.2 \\
\hline Secundaria incompleta & 38 & 9.9 \\
\hline Educación universitaria posgrado completa & 36 & 9.4 \\
\hline Educación universitaria pregrado incompleta & 25 & 6.5 \\
\hline Educación técnica incompleta & 14 & 3.6 \\
\hline Educación universitaria posgrado incompleta & 9 & 2.3 \\
\hline Total & 385 & 100 \\
\hline
\end{tabular}

Fuente: Elaboración propia

Tabla 2. Características de la población en relación con su área de actividad laboral

\begin{tabular}{|l|c|c|}
\hline Área de actividad laboral & $\mathbf{N}$ & $\%$ \\
\hline Ambulante & 107 & 27.8 \\
\hline Sin trabajo & 59 & 15.3 \\
\hline Independiente & 52 & 13.5 \\
\hline Ama de casa & 47 & 12.2 \\
\hline Salud & 35 & 9.0 \\
\hline Otros & 25 & 6.5 \\
\hline Ventas & 14 & 3.6 \\
\hline Alimentaria & 13 & 3.8 \\
\hline Administración & 10 & 2.6 \\
\hline Belleza & 9 & 2.3 \\
\hline Docencia & 7 & 1.7 \\
\hline Limpieza & 7 & 1.7 \\
\hline Total & 385 & 100 \\
\hline
\end{tabular}

Fuente: Elaboración propia 
Tabla 3. Características del tipo de familia con quien convive

\begin{tabular}{|l|c|c|}
\hline Tipo de familia & $\mathbf{N}$ & $\%$ \\
\hline Monoparental & 179 & 46.5 \\
\hline Familia nuclear & 85 & 22.1 \\
\hline Nuclear con parientes próximos & 43 & 11.2 \\
\hline Ampliada & 35 & 9.0 \\
\hline Extensa & 15 & 3.9 \\
\hline Vivo solo & 15 & 3.9 \\
\hline Otros & 7 & 1.8 \\
\hline Nuclear & 4 & 1.0 \\
\hline Reconstituida & 1 & 0.3 \\
\hline Sin familia & 1 & 0.3 \\
\hline Total & 385 & 100 \\
\hline
\end{tabular}

Fuente: Elaboración propia

Tabla 4. Cantidad de personas con las que vive en casa

\begin{tabular}{|l|c|c|}
\hline Cantidad de personas con las que vive en casa & N & \% \\
\hline 2 & 98 & 25.5 \\
\hline 3 & 80 & 20.8 \\
\hline 4 & 67 & 17.4 \\
\hline 1 & 47 & 12.2 \\
\hline 5 & 42 & 10.9 \\
\hline 7 o más & 25 & 6.5 \\
\hline 6 & 17 & 4.4 \\
\hline Solo & 9 & 2.3 \\
\hline Total & 385 & 100 \\
\hline
\end{tabular}

Fuente: Elaboración propia

Tabla 5. Tiempo que lleva viviendo en Perú

\begin{tabular}{|l|c|c|}
\hline Cantidad de años de residencia & $\mathbf{N}$ & $\mathbf{\%}$ \\
\hline Entre 1 año y 2 años & 216 & 56.1 \\
\hline Entre 2 años y 3 años & 102 & 26.5 \\
\hline Menos de 1 año & 51 & 13.3 \\
\hline Entre 3 años y 4 años & 12 & 3.1 \\
\hline Entre 4 años y 5 años & 4 & 1.0 \\
\hline Total & 385 & 100 \\
\hline
\end{tabular}

Fuente: Elaboración propia 
Tabla 6. Tipo de documento que posee

\begin{tabular}{|l|c|c|}
\hline Tipo de documento que posee & $\mathbf{N}$ & $\mathbf{\%}$ \\
\hline PTP (activo o vencido) & 131 & 34.0 \\
\hline Carné de extranjería (o pasaporte con visa humanitaria) & 92 & 23.9 \\
\hline Otro & 73 & 19.0 \\
\hline Carné de solicitante de refugio (o en trámite) & 72 & 18.7 \\
\hline No deseo responder & 17 & 4.4 \\
\hline Total & 385 & 100 \\
\hline
\end{tabular}

Fuente: Elaboración propia

Tabla 7. Asistencia a un centro de salud

\begin{tabular}{|l|c|c|}
\hline En los últimos tres meses, se atendió en un centro médico de tipo & N & $\%$ \\
\hline Ninguno & 274 & 71.2 \\
\hline Atención ambulatoria (postas particulares, clínicas, hospital de la solidaridad) & 58 & 15.0 \\
\hline Seguro Integral de Salud (SIS) & 35 & 9.1 \\
\hline Otro & 9 & 2.3 \\
\hline EsSalud & 6 & 1.6 \\
\hline Seguro privado (EPS) & 3 & 0.8 \\
\hline Total & 385 & 100 \\
\hline
\end{tabular}

Fuente: Elaboración propia

Las tablas muestran las características sociodemográficas identificadas en la población estudiada: la Tabla 1 da cuenta de que $47.8 \%$ de las encuestadas había asistido a la universidad —independientemente de si acabó o no-; en la Tabla 2 vemos que al menos $41.3 \%$ de la muestra trabajaba como ambulante o independiente, es decir, tienen trabajos del sector servicios que han sido justamente los más afectados (Seminario et al., 2020, párr. 5-10); la Tabla 3 permite notar que casi la mitad de las encuestadas $(46.5 \%)$ convive únicamente con sus hijos; y la Tabla 4 caracteriza los hogares de ellas, al mostrar que $46.3 \%$ convive con 2 o 3 personas.

La Tabla 5 indica que el $82.6 \%$ de las encuestadas tiene menos de tres años viviendo en el Perú. Igualmente, $57.9 \%$ tiene un PTP, carné de extranjería o visa humanitaria activo o vencido (Tabla 6). En relación con su salud (Tabla 7), es importante notar que únicamente $11.5 \%$ ha sido atendido con un seguro de salud en los últimos tres meses. 
Tabla 8. Correlaciones significativas entre impacto emocional y efecto de los medios de información

\begin{tabular}{|c|c|c|c|c|c|c|c|}
\hline & Estados en & ionales & & & & & \\
\hline Medios de información & Tranquilo & $\begin{array}{l}\text { Preocu- } \\
\text { pado }\end{array}$ & Temeroso & Aterrado & Enojado & $\begin{array}{l}\text { Desorien- } \\
\text { tado }\end{array}$ & $\begin{array}{l}\text { Emocio- } \\
\text { nado }\end{array}$ \\
\hline Televisión & 0.08 & 0.00 & 0.53 & 0.57 & 0.40 & 0.11 & 0.66 \\
\hline Periódicos & 0.01 & 0.00 & 0.02 & 0.03 & 0.04 & 0.02 & 0.15 \\
\hline Radio & 0.00 & 0.00 & 0.00 & 0.00 & 0.11 & 0.09 & 0.50 \\
\hline Centro de trabajo & 0.00 & 0.00 & 0.02 & 0.00 & 0.15 & 0.02 & 0.03 \\
\hline $\begin{array}{l}\text { Mensajes y conferencias } \\
\text { de prensa del presiden- } \\
\text { te Vizcarra }\end{array}$ & 0.00 & 0.00 & 0.00 & 0.00 & 0.01 & 0.01 & 0.01 \\
\hline Jefes de trabajo & 0.00 & 0.02 & 0.10 & 0.03 & 0.26 & 0.04 & 0.11 \\
\hline $\begin{array}{l}\text { Grupos de WhatsApp } \\
\text { con familiares o com- } \\
\text { pañeros de trabajo }\end{array}$ & 0.30 & 0.00 & 0.05 & 0.20 & 0.04 & 0.82 & 0.74 \\
\hline $\begin{array}{l}\text { Grupos de WhatsApp } \\
\text { con amigos }\end{array}$ & 0.01 & 0.00 & 0.04 & 0.02 & 0.62 & 0.48 & 0.11 \\
\hline Twitter & 0.03 & 0.01 & 0.00 & 0.00 & 0.08 & 0.01 & 0.26 \\
\hline Facebook & 0.00 & 0.00 & 0.00 & 0.00 & 1.00 & 0.12 & 0.98 \\
\hline Mensajes de texto & 0.03 & 0.01 & 0.16 & 0.03 & 0.32 & 0.00 & 0.60 \\
\hline
\end{tabular}

Fuente: Elaboración propia

Tabla 9. Correlaciones significativas entre impacto emocional y cumplimiento de las recomendaciones sanitarias, efecto de los medios de información

\begin{tabular}{|l|c|c|c|c|c|c|c|}
\hline \multicolumn{7}{|c|}{ Estados emocionales } \\
\hline $\begin{array}{l}\text { Recomendaciones } \\
\text { sanitarias }\end{array}$ & $\begin{array}{c}\text { Tran- } \\
\text { quilo }\end{array}$ & $\begin{array}{c}\text { Preocu- } \\
\text { pado }\end{array}$ & $\begin{array}{c}\text { Teme- } \\
\text { roso }\end{array}$ & Aterrado & Enojado & Desorientado & Emocionado \\
\hline $\begin{array}{l}\text { Cumplimiento del } \\
\text { aislamiento social } \\
\text { obligatorio }\end{array}$ & 0.88 & 0.46 & 0.62 & 0.12 & 0.33 & 0.54 & 0.56 \\
\hline $\begin{array}{l}\text { Cumplimiento del lava- } \\
\text { do frecuente de manos } \\
\text { con agua y jabón por } \\
\text { 20 segundos }\end{array}$ & 0.62 & 0.38 & 0.59 & 0.05 & 0.43 & 0.53 & 0.83 \\
\hline $\begin{array}{l}\text { Cubrirse la nariz y la } \\
\text { boca con el antebrazo } \\
\text { o pañuelo desechable } \\
\text { al toser }\end{array}$ & 0.30 & 0.02 & 0.02 & 0.00 & 0.03 & & \\
\hline
\end{tabular}

Fuente: Elaboración propia 
Tabla 10. Correlaciones significativas entre efecto de los medios de información y cumplimiento de las recomendaciones sanitarias

\begin{tabular}{|c|c|c|c|}
\hline & \multicolumn{3}{|c|}{ Cumplimiento de las recomendaciones sanitarias } \\
\hline Medios de información & $\begin{array}{c}\text { Cumplimiento del } \\
\text { aislamiento social } \\
\text { obligatorio }\end{array}$ & $\begin{array}{l}\text { Cumplimiento del } \\
\text { lavado frecuente } \\
\text { de manos con agua } \\
\text { y jabón por } 20 \\
\text { segundos }\end{array}$ & $\begin{array}{c}\text { Cubrirse la nariz y la boca } \\
\text { con el antebrazo o pañuelo } \\
\text { desechable al toser }\end{array}$ \\
\hline Televisión & 0.06 & 0.73 & 0.91 \\
\hline Periódicos & 0.19 & 0.97 & 0.12 \\
\hline Radio & 0.60 & 0.49 & 0.84 \\
\hline Centro de trabajo & 0.13 & 0.65 & 0.00 \\
\hline $\begin{array}{l}\text { Mensajes y conferencias de prensa } \\
\text { del presidente Vizcarra }\end{array}$ & 0.51 & 0.41 & 0.20 \\
\hline Jefes de trabajo & 0.00 & 0.25 & 0.93 \\
\hline $\begin{array}{l}\text { Grupos de WhatsApp con familiares } \\
\text { o compañeros de trabajo }\end{array}$ & 0.77 & 0.21 & 0.09 \\
\hline Grupos de WhatsApp con amigos & 0.97 & 0.38 & 0.10 \\
\hline Twitter & 0.01 & 0.89 & 0.81 \\
\hline Facebook & 0.82 & 0.47 & 0.08 \\
\hline Mensajes de texto & 0.08 & 0.79 & 0.34 \\
\hline
\end{tabular}

Fuente: Elaboración propia

En el nivel correlacional, los hallazgos principales demuestran que existe una correlación positiva débil entre las variables impacto emocional y efecto de los medios de información (22.3\%) con una asociación significativa ( $\mathrm{p}=0.00)$. Lo mismo sucede con la correlación entre las variables impacto emocional y cumplimiento de las recomendaciones sanitarias $(-11.78 \%)$, la cual es negativa y significativa $(\mathrm{p}=0.02)$. Sin embargo, no existe una correlación entre las variables efecto de los medios de información y cumplimiento de las recomendaciones sanitarias $(\mathrm{p}=0.46)$. Esto coincide con los planteamientos de Ponce de León y colaboradores (2010, p. 13), para quienes las variables señaladas no están correlacionadas.

$\mathrm{Al}$ revisar en detalle las correlaciones entre las dimensiones de las variables correlacionadas se puede observar que estas son importantes al tomar en cuenta el impacto emocional y los efectos de los medios de información (Tabla 8). Sin embargo, no ocurre lo mismo al analizar las correlaciones entre las dimensiones de estas variables y las relacionadas con el cumplimiento de las recomendaciones sanitarias (Tabla 9 y Tabla 10).

Con base en lo evidenciado en las tablas se destaca que cada uno de los medios de información (bien sean los medios masivos, como la radio o la televisión, o las redes sociales) están asociados al estado emocional de las mujeres venezolanas migrantes que residen en Lima, principalmente se observa que los mensajes del presidente Martín Vizcarra están más relacionados con todos los estados emocionales analizados; 
así como también que el estado de preocupación de estas mujeres tiene relación con todos los medios informativos estudiados.

Por el contrario, existen pocas correlaciones significativas entre los medios de información y el cumplimiento de las recomendaciones sanitarias; así como entre este último y el impacto emocional. Para el primer caso, solo se destaca que el impacto de la información brindada por la radio tiene una relación débil con el toser o estornudar cubriéndose o tapándose la boca, mientras que los mensajes aparecidos en Twitter también están asociados al cumplimiento del lavado de manos con agua y jabón por al menos 20 segundos; mientras para el segundo caso, únicamente el toser o estornudar cubriéndose o tapándose la boca tiene impacto en las emociones relacionadas con preocupación, temor, miedo y enojo.

Al observar los datos desagregados por cada variable estudiada se obtienen hallazgos importantes. En primer lugar, cuando las mujeres migrantes venezolanas que residen en Lima refieren el impacto que tuvieron en ellas las informaciones brindadas por los medios de comunicación, sus redes sociales y los otros medios informativos se destaca que todos estos espacios comunicativos tuvieron un efecto inquietante en ellas; es decir, la información que diariamente emiten los círculos cercanos a este grupo de población no genera tranquilidad en ellas, sino todo lo contrario.

Si se toma en cuenta únicamente a los medios de comunicación tradicionales (televisión, periódicos y radio), el impacto informativo en las mujeres migrantes venezolanas es alto: todas manifiestan sentirse inquietadas por estos medios $(86.7 \%$, $73.5 \%$ y $61.3 \%$, respectivamente).

De igual forma, las mujeres migrantes venezolanas que residen en Lima dan cuenta de que los mensajes enviados a través de las redes sociales (Facebook y Twitter), así como por los grupos de WhatsApp, han tenido, tal como los medios de comunicación, un impacto principalmente inquietante en ellas: $87 \%$ de los grupos de WhatsApp con familiares y compañeros de trabajo (para quienes cuentan con uno) generó información inquietante, $85 \%$ de los grupos únicamente con amigos en esta aplicación sintió que los mensajes recibidos eran igualmente de este tipo; así como $81 \%$ de los contenidos que observaron aquellas que cuentan con una cuenta en Facebook fueron también inquietantes. Este fenómeno también ocurrió en aquellas que observaron la información de Twitter (de 48\% del total que accedió a esta red social, 94\% halló esta información inquietante).

De igual forma, al notar el impacto de las informaciones de las autoridades peruanas, es relevante mencionar que se tomaron en cuenta dos canales: las conferencias de prensa y declaraciones del presidente Martín Vizcarra, además de los mensajes de texto. Sobre este último, es importante observar que el Estado peruano tuvo una política de envío de mensajes de ayuda e información a todos aquellos con un móvil registrado en territorio peruano.

Para las mujeres venezolanas migrantes, sin embargo, estos dos canales tuvieron un efecto principalmente inquietante sobre ellas: $70 \%$ para el primero y $56 \%$ para el segundo. Vale notar que, para quienes pertenecen a un entorno laboral estable, $82 \%$ de las informaciones enviadas en este entorno también fueron inquietantes.

Los datos revelados en la encuesta permiten determinar que, más allá de los medios de comunicación, los propios espacios de socialización digital de las mujeres migrantes venezolanas están generando inquietud. Estos espacios están compuestos en su mayoría por quienes conforman sus círculos más cercanos: por un lado, todos aquellos a quienes se dejó en el país natal, los familiares y amigos que residen en el 
lugar de origen; por otro, los amigos, conocidos, compañeros de trabajo y familiares que también migraron y radican en el lugar de destino.

Al referir el impacto de las redes sociales digitales en tanto información, se ha dejado de lado el impacto emocional que generan no solo estas, sino también los medios de comunicación y los mensajes estatales. Las mujeres venezolanas migrantes encuestadas para este estudio afirman tener una alta preponderancia de sentimientos tales como preocupación (muchísimo 39\% y mucho 41\%), miedo (muchísimo 27\% y mucho $36 \%$ ), terror (muchísimo $20 \%$ y mucho $21 \%$ ); es decir, emociones que implican altos niveles de estrés en ellas. De igual forma, admiten sentirse entre nada y muy poco emocionadas ( $54 \%$ y $16 \%$, respetivamente); nada y poco enojadas (35\% y $19 \%$, respectivamente) e igualmente poco y nada desorientadas (23\%, en ambos casos). Finalmente, el sentimiento de tranquilidad, que ya había sido referido en relación con la información de los medios informativos, muestra que el impacto emocional en ellas ha tenido un efecto relevante: $31 \%$ se siente muy poco tranquilas; mientras $28 \%$, nada tranquilas.

Sobre la tercera variable de análisis es relevante considerar que las mujeres venezolanas migrantes cumplieron siempre y casi siempre las recomendaciones sanitarias recomendadas por el gobierno peruano y por la oms: mantuvieron el aislamiento social obligatorio ( $70 \%$ y $26 \%$, respectivamente), se lavaron las manos con agua y jabón por al menos 20 segundos ( $84 \%$ y $12 \%$, respectivamente) y se cubrieron la nariz y boca con un pañuelo o el antebrazo al estornudar (92\% y $5 \%$, respectivamente).

\section{Discusión y conclusiones}

Los mensajes enviados por las autoridades peruanas no pudieron dar tranquilidad a las mujeres venezolanas residentes en Lima en este contexto. Esto reafirma lo observado por Luzes y colaboradores (2020, p. 2) del Centro de Investigaciones de la Universidad del Pacífico, quienes indican que las políticas públicas para reducir el impacto del COVID-19 en las poblaciones vulnerables no ha contemplado a la población migrante venezolana: ellos no fueron incluidos dentro del bono para personas en estado de vulnerabilidad del gobierno peruano, tampoco dentro del bono para las personas que trabajan sin un contrato laboral, e incluso tras el reparto de algunas canasta básicas con alimentos, muchos gobiernos locales decidieron no incluir a la población migrante; finalmente tampoco se ha contemplado el caso de las familias que alquilan una vivienda, ya que ninguna medida concreta ha solucionado este tema. En este contexto, la información que las autoridades brindaron no consiguió tener un efecto positivo en estas.

En el contexto del COVID-19 en Perú, el consumo de los medios de comunicación tradicionales ha incrementado (Instituto de Estudios Peruanos [IEP], 2020, p. 4); no obstante, los mensajes enviados por los medios de comunicación tampoco consiguieron tener un efecto de tranquilidad en las mujeres venezolanas migrantes, por el contrario, les resultaron principalmente inquietantes. Este dato es relevante en la medida en que diversos estudios demuestran que los medios de comunicación son importantes en la conformación de espacios de representación social del total de la sociedad de una manera plural y democrática (Angulo-Giraldo et al., 2020, p. 397; Lario, 2006, p. 41; Masanet Ripoll \& Ripoll Arcacia, 2008, p. 183); y, en relación con la población migrante, 
son importantes tanto como escenarios que contribuyen en el tránsito de los propios migrantes hacia los lugares de residencia (Echeverría Victoria, 2013, p. 68), así como en la reconstrucción del imaginario de las relaciones sociales que estos establecen en los lugares a los que arriban (Castilla-Vázquez, 2017, p. 149).

El sentimiento de inquietud mostrado por las mujeres migrantes venezolanas ante la información brindada por la radio, la televisión y la prensa debe considerarse en relación con tres contextos mediáticos: las narraciones sobre los migrantes que influyen en un sentimiento de exclusión y expulsión hacia estos; ${ }^{6}$ las representaciones de las migrantes mujeres en las que estas son victimarias o sujetos pasivos; ${ }^{7} \mathrm{y}$, en el caso específico del grupo de estudio, una categorización de estas como trágicas, dramáticas, delictivas o quienes quitan oportunidades laborales. ${ }^{8}$

Así, el sentimiento de inquietud por la información recibida de estos medios de comunicación puede deberse no solo al rechazo hacia la información contextual referida a la pandemia y su manejo, sino a un relato histórico reciente en el que son estos medios los que han construido y reconstruido estereotipos y prejuicios sobre los migrantes (Masanet Ripoll \& Ripoll Arcacia, 2008, p. 173) y en los cuales ellas, además, han sido subrepresentadas o vistas como sujetos pasivos o secundarios. A partir de lo anterior, queda pendiente analizar el rol de la prensa en contextos de crisis social, y considerar esencial no promover una alarma social, no incrementar la percepción del riesgo, sino por el contrario, contribuir en la reducción de la ansiedad y el pánico (Jurado Salván \& Jurado Izquierdo, 2014, p. 99; Lázaro-Rodríguez \& Herrera-Viedma, 2020, p. 8).

Más allá de los espacios mediáticos tradicionales, cabe observar en detalle los espacios más íntimos de socialización de las mujeres migrantes venezolanas, es decir, las redes sociales digitales. Si bien la importancia de estos espacios de socialización digital ya ha sido demostrada en investigaciones precedentes (Aire Laureano, 2017, p. 75; Komito, 2011, p. 1081; Komito \& Bates, 2011, p. 292; Larsen et al., 2008, p. 430; Lin, 1986, p. 55; Ogáyar-Marín et al., 2018, p. 112), para el caso específico de las mujeres migrantes venezolanas cobra mayor relevancia, puesto que estudios han mostrado que los comentarios negativos sobre ellas en redes como Facebook les generaron sensaciones de inseguridad y rechazo (Velarde Ramirez, 2019, pp. 11-12; Blouin, 2019, pp. 88-89; Mesa Reina, 2020, p. 63).

En el Perú, el consumo de redes sociales digitales como Facebook es de cerca de cuatro horas (Ipsos, 2019, p. 1), a lo cual se agrega que durante la pandemia

\footnotetext{
${ }^{6}$ Se han construido dos escenarios hacia los migrantes: el primero, derivado de las élites, en el que la voz de los migrantes es siempre una voz pasiva o una fuente secundaria y donde se les categoriza como un "problema" a ser excluido y rechazado; el segundo, que proviene de los sectores populares, en que se muestra un intento por excluir y alejar a los migrantes (Martínez, 2008, p. 25; Ordóñez \& Ramírez Arcos, 2019, p. 64; Sar, 2016, p. 30; Vázquez-Aguado, 1999, p. 58).

${ }^{7}$ Las representaciones mediáticas se acentúan al considerar la variable de género. Así, las mujeres migrantes son clasificadas en dos sentidos: victimarias que trasgreden las normas sociales de los lugares a donde han migrado; o sujetos pasivos que padecen las acciones de un victimario (Masanet Ripoll \& Ripoll Arcacia, 2008, p. 170; Ordóñez \& Ramírez Arcos, 2019, p. 64; Van Dijk, 2008, p. 13-14).

${ }^{8}$ Los medios de comunicación peruanos han construido una representación parcial de estas caracterizadas de tres formas: las trágicas y dramáticas que hacen "parte de familias numerosas con varios hijos" y las "mujeres embarazadas"; las delictivas; y aquellas que amenazan a lo local, al argüir que son "una competencia desigual para el trabajador local” (Mesa Reina, 2020, p. 170).
} 
el consumo a través de redes fijas (dentro de casa) como WhatsApp y Facebook se incrementó en 184\% y 177\%, respectivamente (Organismo Superior de Inversión Privada en telecomunicaciones [Osiptel], 2020, p. 1). En ese contexto de alto uso, los espacios de socialización digital tampoco permitieron que las mujeres migrantes venezolanas recibieran mensajes de calma y tranquilidad: tanto los grupos de WhatsApp con amigos, familiares y entornos laborales, así como el propio Facebook fueron escenarios de difusión de informaciones inquietantes.

Así, además de las afectaciones producidas por la información compartida desde los medios de comunicación, las redes sociales digitales —el entorno familiar, amical y laboral más íntimo- también tienen un impacto negativo al generar inquietud (y no tranquilidad) en las mujeres venezolanas migrantes (Ogáyar-Marín et al., 2018, p. 113).

En ese escenario, el estado emocional propio de estas mujeres se ve afectado triplemente: no solo por sus condiciones sociodemográficas anteriormente descritas (que sean mujeres, que sean migrantes, que pertenezcan principalmente al sector laboral que trabaja en la informalidad y que estén en situación de pobreza [Ramírez Lasso, 2018, p. 47]), sino porque en un contexto de crisis mundial y nacional, su propio contexto local les genera mayor estrés y contribuye poco a mejorar esta situación. En ese sentido, las afectaciones en la salud mental de las mujeres venezolanas migrantes coinciden con lo hallado en casos como Jordania, donde las mujeres se han visto más afectadas que los hombres en su bienestar mental y carga económica (Abufaraj et al., 2021).

El estrés derivado del propio proceso migratorio (Millán-Franco et al., 2019, p. 123) afecta los estilos de vida, los valores y la dignidad de las personas, todo lo cual favorece la "presencia de estados emocionales que afectan la salud mental" (Torres López et al., 2014, párr. 8), lo cual se combina negativamente con los altos índices de preocupación, temor, miedo y enojo que manifiestan ellas; por lo que se hace necesario el establecimiento de políticas públicas que permitan la construcción de mecanismos de apoyo social para ellas.

De igual forma, al observar la alta incidencia de emociones que afectan la salud mental de las mujeres migrantes, sobre todo en el contexto de la pandemia por la COVID-19, se hace relevante profundizar en las características de las redes de apoyo social (familia, amigos, entre otros), tanto de los connacionales del migrante, como del grupo de acogida, dado que la existencia de estas redes "provided invaluable 'stressbuffering' effects" (McKay et al., 2003, p. 13). Por ello, el reconocer la importancia de estas redes invita a reflexionar sobre el rol que ejercen tanto los medios informativos, como las mismas redes sociales digitales en tanto entornos laborales y amicales que comparten mensajes hacia ellas.

Si bien las dos variables analizadas anteriormente no han demostrado tener una correlación significativa con el correcto cumplimiento de las recomendaciones sanitarias (tercera variable) que llevaron a cabo las mujeres venezolanas migrantes. En esa línea, los estudios demuestran que los lazos y las relaciones sociales influyen positivamente en la salud de los migrantes (Alegría et al., 2017, p. 149), de manera que, en la medida en que las relaciones sociales son menores, aumentan los problemas de depresión o ansiedad (Kiang et al., 2020, p. 386); por ello, la ausencia de redes de apoyo que proporcionen moderadas ventajas de salud (Ruiz et al., 2016, p. 470) las colocan en una mayor vulnerabilidad. Lo anterior se debe tomar en cuenta para futuros estudios y para el desarrollo de políticas públicas. 
En contraste a la ausencia de correlación presentada en esta investigación, recientes estudios encuentran que las restricciones de permanencia en el hogar impactan en la salud mental (depresión, ansiedad, insomnio, estrés) respecto a otras poblaciones (Killgore et al., 2021), lo cual se agrava para las personas en un estado de mayor vulnerabilidad pues reciben un mayor impacto en su salud mental, así como sufren más las restricciones de movilización o aislamiento social (Kim \& Laurence, 2020). Por ello, queda pendiente la incidencia en mayor profundidad sobre el establecimiento de las afectaciones en las mujeres migrantes venezolanas.

\section{Agradecimientos}

Los autores del estudio agradecen el apoyo de Karina Núñez Paz, de la ong Unión Venezolana y de la Asociación Pasos Firmes; así como del profesor Felix Colina Ysea.

\section{Referencias}

Abufaraj, M., Eyadat, Z., Al-Sabbagh, M. Q., Nimer, A., Moonesar, I. A., Yang, L., Al Khatib, W. \& Al-Qutob, R. (2021). Gender-based disparities on health indices during COVID-19 crisis: a nationwide cross-sectional study in Jordan. International Journal for Equity in Health, 20, artículo 91, 1-10. https:/ / doi.org/10.1186/ s12939-021-01435-0

Adewunmi, M., Akintelu, S. O., Oladele, W. \& Taiwo, O. (2021). Exploratory Data Analysis (EDA) of social media impact on Lagos residents during COVID-19 pandemic. Sau Journal of Management and Social Sciences, 6(1), 164-178. https:// journals.sau.edu.ng/index.php/sjmas/article/view/249/174

Aguirre, S. (2020, 8 de abril). Las dudas que dejó el segundo paquete de medidas económicas del gobierno. GNN Chile. https://www.cnnchile.com/coronavirus/dudas-segundo-paquete-medidas-economicas-gobierno_20200408/

Aire Laureano, K. (2017). Migração e identidade. Um estudo sobre os andinos no Rio de Janeiro (Tesis de maestria, Universidad Federal de Río de Janeiro]. http://www.pos. eco.ufrj.br/site/download.php?arquivo=upload/disserta_klaureano_2017.pdf

Akingbade, O. (2018, octubre). Epidemics, fears and the mass media: an analysis of the 2014 ebola virus disease outbreak in Nigeria. Journal of Communication and Media Research, 10(2), 139-148. https:/ /www.academia.edu/38748646/Epidemics_Fears_and_the_Mass_Media_An_Analysis_of_the_2014_Ebola_Virus_Disease_Outbreak_in_Nigeria._OLUTOBI_AKINGBADE?auto=download

Alegría, M., Álvarez, K. \& DiMarzio, K. (2017, junio). Immigration and mental health. Current epidemiology reports, 4(2), 145-155. https://doi.org/10.1007/s40471-0170111-2

Alto Comisionado de las Naciones Unidas para los Refugiados (ACNUR). (2020). Plataforma de coordinación para refugiados y migrantes de Venezuela. Data2unhcr. https:// data2.unhcr.org/es/situations/platform 
Ammar, A., Brach, M., Trabelsi, K., Chtourou, H. Boukhris, O., Masmoudi, L., Bouaziz, B., Bentlage, E., How, D., Ahmed, M., Müller, P., Müller, N., Aloui, A., Hammouda, O., Paineiras-Domingos, L. L., Braakman-Jansen, A., Wrede, C., Bastoni, S., Soares Pernambuco, C., ... Hoekelmann, A. (2020). Effects of COVID-19 home confinement on eating behaviour and physical activity: results of the ECLBCOVID19 international online survey. Nutrients, 12(6). https://pubmed.ncbi. nlm.nih.gov/32481594/

Angulo-Giraldo, M. \& Bolo-Varela, O. (2021). Medios de comunicación y conflictos sociales durante la pandemia por COVID-19: análisis de los enfoques presentes en la prensa de Lima durante las protestas contra el gobierno interino de Manuel Merino (2020). Desde el Sur, 13(1), e0005. https://doi.org/10.21142/DES-13012021-0005

Angulo-Giraldo, M. A., Ramos Bonilla, G., Chamorro García, H., Colonia, L. \& Fernandez-Alvarado, S. (2020). Personas mayores en los medios digitales peruanos durante la pandemia por COVID-19. Revista Kairós: Gerontologia, 23(núm. especial 28), 391-416. https://revistas.pucsp.br/index.php/kairos/article/view/51532

Blouin, C. (Coord.). (2019). Después de la llegada: realidades de la migración venezolana. Pontificia Universidad Católica del Perú/Editorial Jurídica THEMIS. https:// repositorio.pucp.edu.pe/index/bitstream/handle/123456789/170254/ Despue\%C3\%8C\%C2\%81s\%20de\%20la\%20llegada.\%20Realidades $\% 20$ de $\% 201 \mathrm{la} \% 20$ migracio $\% \mathrm{C} 3 \% 8 \mathrm{C} \% \mathrm{C} 2 \% 81 \mathrm{n} \% 20$ venezolana.pdf?sequence $=1 \&$ \&isAllowed $=y$

Brooks, S. K., Weston, D. \& Greenberg, N. (2020). Psychological impact of infectious disease outbreaks on pregnant women: Rapid evidence review. MedRxiv. https://doi. org/10.1101/2020.04.16.20068031

Castilla-Vázquez, C. (2017). Mujeres en transición: la inmigración femenina africana en España. Migraciones internacionales, 9(33), 143-171. https://doi.org/10.17428/ rmi.v9i33.290

Cisneros, J. D. (2008, invierno). Contaminated communities: the metaphor of "immigrant as pollutant" in media representations of immigration. Rhetoric and Public Affairs, 11(4), 569-601. https://www.jstor.org/stable/41940396

Cogo, D. (2014). Los estudios de recepción en América Latina: perspectivas teórico-metodológicas. Portal de la Comunicación InCom-UAB. https:/ /incom.uab.cat/ portalcom/wp-content/uploads/2020/01/48_esp.pdf

Durkin, S. \& Wakefield, M. (2008). Interrupting a narrative transportation experience: program placement effects on responses to antismoking advertising. Journal of Health Communication, 13(7), 667-680. https://doi. org/10.1080/10810730802412248

Echeverría Victoria, M. (2013, enero-junio). Cultura migratoria y comunicación masiva e interpersonal en los imaginarios juveniles. Comunicación y sociedad, (19), 61-86. http://www.scielo.org.mx/scielo.php?script=sci_arttextypid $=$ S0188-252X2013000100004

Eggener, S. (1998). The power of the pen: medical journalism and public awareness. JAMA, 279(17), 1400. https://doi.org/10.1001/jama.279.17.1400-JMS0506-4-0

Ekman, P. \& Friesen, W. (1978). Facial action coding systems. Consulting Psycologists Press. 
Fleury, A. (2016, febrero). Understanding women and migration: A literature review (Knomad Working Paper 8). Federal Ministry for Economic Cooperation and Development/Deutsche Gesellschaft für Internationale Zusammenarbeit/Government Offices of Sweden/Swiss Agency for Development and Cooperation SDC/ World Bank Group. http://atina.org.rs/sites/default/files/KNOMAD\%20Understaning\%20Women\%20and\%20Migration.pdf

Fowks, J. (2020). Perú aplica el plan económico más ambicioso de la región para enfrentar la pandemia. El País. https://elpais.com/economia/2020-04-04/peru-aplica-el-plan-economico-mas-ambicioso-de-la-region-para-enfrentar-la-pandemia.html

Goleman, D. \& Cherniss, C. (2013). Inteligencia emocional en el trabajo: cómo seleccionar y mejorar la inteligencia emocional en individuos, grupos y organizaciones. Editorial Kairós.

Guanipa Ramírez, L. F. (2019). Las personas y las organizaciones: el equilibrio laboral. Emprende y Transforma, 1(1), 29-38. https://www.researchgate.net/publication/335966001_Las_personas_y_las_organizaciones_el_equilibrio_laboral_ People_and_organizations_the_work-life_balance

Gutiérrez San Miguel, B., Rodríguez Fidalgo, M. I. \& Gallego, M. C. (2010). El papel de los medios de comunicación actuales en la sociedad contemporánea española. Signo y pensamiento, 29(57), 268-285. https://www.redalyc.org/ pdf/860/86020052017.pdf

Hopenhayn, M. \& Bello, A. (2001, mayo). Discriminación étnico-racial y xenofobia en América Latina y el Caribe (Serie Políticas Sociales núm. 47). Cepal. https://repositorio.cepal.org/bitstream/handle/11362/5987/1/S01050412_es.pdf

Infomed-Centro Nacional de Información en Ciencias Médicas. (2020, 13 de octubre). Actualización epidemiológica de la situación del coronavirus (COVID-19) en el mundo. Vigilancia en salud pública. https://temas.sld.cu/vigilanciaensalud/2020/10/09/actualizacion-epidemiologica-de-la-situacion-del-coronavirus-covid-19-en-el-mundo-120/

Instituto de Estudios Peruanos (IEP). (2020, 26 de abril). Informe de opinión-abril 2020. COVID-19 y medios de comunicación. Encuesta telefónica. https://iep.org.pe/ wp-content/uploads/2020/04/Informe-OP-Abril-2020-5-Medios-de-comunicaci\%C3\%B3n-y-Covid-19-2.pdf

Instituto Nacional de Estadística e Informática (INEI). (2018). Condiciones de vida de la población venezolana que reside en Perú. Resultados de la "Encuesta dirigida a la población venezolana que reside en el país". https:/ /www.inei.gob.pe/media/MenuRecursivo/publicaciones_digitales/Est/Lib1666/

Ipsos. (2019). El 55\% del tiempo de consumo aún ocurre en medios tradicionales. https:// www.ipsos.com/es-pe/el-55-del-tiempo-de-consumo-aun-ocurre-en-medios-tradicionales

Irvine, M., Coombs, D., Skarha, J., del Pozo, B., Rich, J., Taxman, F. \& Green, T. C. (2020). Modeling COVID-19 and its impacts on us Immigration and Customs Enforcement (ICE) Detention Facilities, 2020. Journal of Urban Health, 97, 439447. https://doi.org/10.1007/s11524-020-00441-x

Issaka, B. (2021). Alienating: how the portrayal of Muslim women in us media affects Muslim women's social identities [Tesis inédita de doctorado, Kansas States University]. 
Jefferson, T., Del Mar, C. B., Dooley, L., Ferroni, E., Al-Ansary, L. A., Bawazeer, G. A., Van Driel, M. L., Nair, N. S., Jones, M. A., Thorning, S. \& Conly, J. M. (2011, 6 de julio). Physical interventions to interrupt or reduce the spread of respiratory viruses. Cochrane Database of Systematic Reviews, (7). https://doi. org/10.1002/14651858.CD006207.pub4

Jernigan, D. B. (2020). Update: public health response to the coronavirus disease 2019 outbreak-United States, February 24, 2020. Morbidity and Mortality Weekly Report, 69(8), 216-219. https://doi.org/10.15585/mmwr.mm6908e1

Jurado Salván, E. \& Jurado Izquierdo, M. (2014). Los errores de comunicación en la crisis del ébola. Cuadernos de periodistas: revista de la Asociación de la Prensa de Madrid, (29), 90-99. http://www.cuadernosdeperiodistas.com/media/2015/02/90-99-SALVAN-Y-JURADO.pdf

Kalfeli, N., Frangonikolopoulos, C. \& Gardikiotis, A. (2020, octubre). Expanding peace journalism: a new model for analyzing media representations of immigration. Journalism. https://doi.org/10.1177/1464884920969089

Kasperson, R. E. \& Kasperson, J. X. (1996). The social amplification and attenuation of risk. The Annals of the American Academy of Political and Social Science, 545(1), 95-105. https://doi.org/10.1177/0002716296545001010

Kasperson, R. E., Renn, O., Slovic, P., Brown, H. S., Emel, J., Goble, R., Kasperson, J. X. \& Ratick, S. (1988). The social amplification of risk: a conceptual framework. Risk Analysis, 8(2), 177-187. https://doi.org/10.1111/j.1539-6924.1988. tb01168.x

Khan, Z., Muhammad, K., Ahmed, A. \& Rahman, H. (2020). Coronavirus outbreaks: prevention and management recommendations. Drugs y Therapy Perspectives, (36), 215-217. https://doi.org/10.1007/s40267-020-00717-x

Kiang, L., Grzywacz, J. G., Marín, A. J., Arcury, T. A. \& Quandt, S. A. (2020). Mental health in immigrants from nontraditional receiving sites. Cultural Diversity and Ethnic Minority Psychology, 16(3), 386-394. https://doi.org/10.1037/a0019907

Killgore, W. D., Cloonan, S. A., Taylor, E. C. \& Dailey, N. S. (2021). Mental health during the first weeks of the COVID-19 pandemic in the United States. Frontiers in Psychiatry, 12, artículo 561898. https://doi.org/10.3389/fpsyt.2021.561898

Kim, H. H. S., \& Laurence, J. (2020). COVID-19 restrictions and mental distress among American adults: evidence from Corona Impact Survey (W1 and W2). Journal of Public Health, 42(4), 704-711. https://pubmed.ncbi.nlm.nih.gov/32880640/

Koechlin, J. \& Eguren, J. (Eds.). (2018). El éxodo venezolano: entre el exilio y la emigración. UARM/Konrad Adenauer Stiftung/orm/Obimid. https://issuu.com/inmrd/ docs/el_xodo_venezolano_entre_la_exilio

Komito, L. (2011). Social media and migration: Virtual community 2.0. Journal of the American Society for Information Science and Technology, 62(6), 1075-1086. https:/ / doi.org/10.1002/asi.21517

Komito, L. \& Bates, J. (2011). Migrants' information practices and use of social media in Ireland: networks and community. Proceedings of the 2011 iConference, (11), 289-295. https://doi.org/10.1145/1940761.1940801

La, V.-P., Pham, T.-H., Ho, M.-T., Nguyen, M.-H., P Nguyen, K.-L., Vuong, T.-T., Nguyen, H.-K.T., Tran, T., Khuc, Q., Ho, M.-T. \& Vuong, Q. H. (2020). Policy response, social media and science journalism for the sustainability of the public health 
system amid the COVID-19 outbreak: The Vietnam lessons. Sustainability, 12(7), artículo 2931. https://doi.org/10.3390/su12072931

Lario, M. (Coord.). (2006). Medios de comunicación e inmigración. Caja de Ahorros del Mediterráneo.

Larsen, J., Urry, J. \& Axhausen, K. (2008). Mobilities, networks, geographies. The Professional Geographer, 60(3), 429-430. https://www.tandfonline.com/doi/citedby $/ 10.1080 / 00330120802115391$ ?scroll=topyneedAccess=true

Lasén Díaz, A. (2014). Introducción. Las mediaciones digitales de la educación sentimental de los y las jóvenes. En: I. Megías Quirós \& E. Rodríguez San Julián, Jóvenes y comunicación. La impronta de lo virtual (pp. 7-16). Centro Reina Sofía sobre Adolescencia y Juventud/Fundación de Ayuda contra la Drogadicción. https://www.adolescenciayjuventud.org/publicacion/jovenes-y-comunicacion-la-impronta-de-lo-virtual/?category_id $=47$

Lázaro-Rodríguez, P. \& Herrera-Viedma, E. (2020). Noticias sobre COVID-19 y 2019nCoV en medios de comunicación de España: el papel de los medios digitales en tiempos de confinamiento. El profesional de la información (EPI), 29(3), 1-11. https://doi.org/10.3145/epi.2020.may.02

Lima, C. K. T., Carvalho, P. M. D. M., Lima, I. D. A. A. S., Nunes, J. V. A. D. O., Saraiva, J. S., de Souza, R. I., da Silva, C. G. L. \& Neto, M. L. R. (2020). The emotional impact of Coronavirus 2019-nCoV (new Coronavirus disease). Psychiatry Research, 287, artículo 112915. https://doi.org/10.1016/j.psychres.2020.112915

Limcaoco, R. S. G., Mateos, E. M., Fernandez, J. M. \& Roncero, C. (2020). Anxiety, worry and perceived stress in the world due to the COVID-19 pandemic, March 2020. Preliminary results. medRxiv. https://doi.org/10.1101/2020.04.03.20043992

Lin, N. (1986). Conceptualizing social support. En N. Lin, A. Dean \& W. Ensel (Eds.), Social support, life events, and depression. Academic Press.

Luzes, M., Freier, F. \& Bird, M. (2020, abril). Propuesta de política pública 5: Salud pública y la población migrante en el Perú. COVID-19 y la importancia de políticas inclusivas. Centro de Investigación de la Universidad del Pacífico. https://ciup.up.edu. pe/media/1602/ciup-ppp-no5.pdf

Ma, J. \& Stahl, L. (2017). A multimodal critical discourse analysis of anti-vaccination information on Facebook. Library $\mathcal{E}$ Information Science Research, 39(4), 303-310. https://doi.org/10.1016/j.lisr.2017.11.005

Maeda Jerí, J. (Coord.). (2021, junio). Boletín n. 2. Percepción sobre la sociedad y la migración venezolana en el Perú. Instituto de Democracia y Derechos Humanos de la Pontificia Universidad Católica del Perú. https://idehpucp.pucp.edu.pe/ lista_publicaciones/boletin-n02-percepcion-sobre-la-sociedad-y-la-migracionvenezolana-en-el-peru/

Marasinghe, K. M. (2020). Concerns around public health recommendations on face mask use among individuals who are not medically diagnosed with COVID-19 supported by a systematic review search for evidence. Research Square. https://doi.org/10.21203/ rs.3.rs-16701/v3

Martínez, M. (2008). Inmigración, discurso y medios de comunicación. Instituto Alicantino de Cultura Juan Gil-Albert. 
Masanet Ripoll, E. \& Ripoll Arcacia, C. (2008). La representación de la mujer inmigrante en la prensa nacional. Papers. Revista de Sociología, 89, 169-185. https:// doi.org/10.5565/rev/papers/v89n0.753

Mboera, L. E. G. \& Rumisha, S. F. (2004). The role of mass media in disease outbreak reporting in the United Republic of Tanzania. Tanzania Journal of Health Research, 6(2), 73-78. https://doi.org/10.4314/thrb.v6i2.14246

McKay, L., Macintyre, S. \& Ellaway, A. (2003). Migration and health: a review of the international literature (Occasional Paper No. 12). MrC Social and Public Health Sciences Unit. http://www.sphsu.mrc.ac.uk/library/occasional/OP012.pdf

Mendoza, W. \& Miranda, J. J. (2019). La inmigración venezolana en el Perú: desafíos y oportunidades desde la perspectiva de la salud. Revista Peruana de Medicina Experimental y Salud Publica, 36(3), 497-503. https://doi.org/10.17843/rpmesp.2019.363.4729

Mesa Reina, S. M. (2020). El migrante venezolano visto desde los medios y las audiencias: un estudio comparativo de la relación entre noticias televisivas y cognición social sobre los venezolanos inmigrantes en el Perú y Colombia [Tesis de maestría, Pontificia Universidad Católica del Perú]. https://tesis.pucp.edu.pe/repositorio/bitstream/handle/20.500.12404/16387/MESA_REINA_SANDRA_MILENA\%20 $\% 281 \% 29$.pdf? sequence $=1$ \&isAllowed $=\mathrm{y}$

Millán-Franco, M., Gómez-Jacinto, L., Hombrados-Mendieta, M. I. \& García-Cid, A. (2019). Las redes de apoyo social online y offline en los inmigrantes de Málaga (España). Migraciones. Revista del Instituto Universitario de Estudios sobre Migraciones, (47), 119-149. https://doi.org/10.14422/mig.i47y2019.005

Ministerio de Salud. (2020, 7 de octubre). Minsa: casos confirmados por coronavirus Covid-19 ascienden a 835662 en el Perú (Comunicado $N^{\circ}$ 264). https://www.gob. $\mathrm{pe} /$ institucion/minsa/noticias/306292-minsa-casos-confirmados-por-coronavirus-covid-19-ascienden-a-835-662-en-el-peru-comunicado-n-264

Moore, H. E. \& Friedsam, H. J. (1959). Reported emotional stress following a disaster. Social Forces, 38(2), 135-139. https://doi.org/10.2307/2573933

Mulsow, G. (2008). Desarrollo emocional: impacto en el desarrollo humano. Educação, 31(1), 61-65. https://www.redalyc.org/pdf/848/84806409.pdf

Ogáyar-Marín, F. J., Muntean, V. \& Gamella-Mora, J. F. (2018). Redes sociales digitales en la migración trasnacional romá de Rumanía. Una "polymedia" transnacional. Revista de humanidades, (35), 107-136. https://dialnet.unirioja.es/servlet/ articulo?codigo $=6624969$

Ordóñez, J. T. \& Ramírez Arcos, H. E. (2019). (Des)orden nacional: la construcción de la migración venezolana como una amenaza de salud y seguridad pública en Colombia. Revista Ciencias de la Salud, 17, 48-68. https://doi.org/10.12804/ revistas.urosario.edu.co/revsalud/a.8119

Organismo Superior de Inversión Privada en Telecomunicaciones (Osiptel). (2020). Uso del whatsapp web crece sostenidamente desde el inicio del aislamiento social. https:// www.osiptel.gob.pe/portal-del-usuario/noticias/uso-de-whatsapp-facebook-ytiktok-crece-sostenidamente-en-las-redes-fijas-de-internet-durante-la-cuarentena/

Organización de las Naciones Unidas (ONU). (2019). Los efectos de la migración en las mujeres y las niñas migrantes: una perspectiva de género. (Informe A/HRC/41/38). https://reliefweb.int/sites/reliefweb.int/files/resources/G1910794.pdf 
Organización Internacional para las Migraciones (оIM). (2020, 5 de junio). Crisis de refugiados y migrantes venezolanos. https://www.iom.int/es/crisis-de-refugiados-y-migrantes-venezolanos

Pastrana Valls, A. (2017). El impacto de la movilidad cognitiva y los medios de información en la participación política de los mexicanos. Cuadernos.info, (40), 17-37. https://doi.org/10.7764/cdi.40.1096

Patel, A. \& Jernigan, D. B. (2020). Initial public health response and interim clinical guidance for the 2019 novel coronavirus outbreak-United States, December 31, 2019-February 4, 2020. Morbidity and Mortality Weekly Report, 69(5), 140-146. https://doi.org/10.15585/mmwr.mm6905e1

Ponce de León, S., Arévalo-Ramírez, M., Santiago-Ventura, Y., González, J. \& Lara, M. (2010). Percepción del riesgo, impacto emocional, efecto de los medios de información y cumplimiento de las recomendaciones sanitarias en la contingencia por influenza A-H1N1. Psiquiatría, 26(2), 8-16.

Portal de Datos Mundiales sobre la Migración. (2021, 16 de julio). Bienvenidos al Portal de Datos Mundiales sobre la Migración. https://migrationdataportal.org/es/ node $/ 607$

Programa de las Naciones Unidas para el Desarrollo (PNUD). (2020). Los impactos económicos del COVID-19 y las desigualdades de género recomendaciones y lineamientos de políticas públicas. https://www.latinamerica.undp.org/content/rblac/es/ home/library/womens_empowerment/los-impactos-economicos-del-covid-19y-las-desigualdades-de-gene.html

Ramírez Lasso, L. M. (2018). Representaciones discursivas de las migrantes venezolanas en medios digitales. Revista Latinoamericana de Estudios del Discurso, 18(2), 42-58. https://raled.comunidadaled.org/index.php/raled/article/view/319

Reguillo, R. (1998). Imaginarios globales, miedos locales: la construcción social del miedo en la ciudad [Ponencia]. IV Encuentro de la Asociación Latinoamericana de Investigadores de la Comunicación. ALAIc. "Ciencias de la comunicación: identidades y fronteras". Universidad Católica de Pernambuco, Recife, Brasil, 11-16 de septiembre de 1998. http://www.mamacoca.org/docs_de_base/La_Representacion_Social_del_narcotrafico/Rossana_Reguillo_Imaginarios_la_construccion_social_del_miedo_en_la_ciudad_ALAIC_11-16_de_septiembre_de_1998. pdf

Rodríguez, M. (2006). La comunicación social en el manejo de los problemas psicosociales en situaciones de emergencia. En: J. Rodríguez, M. Zacarreli \& R. Pérez (Eds.), Guía práctica de salud mental en situaciones de desastre (pp. 169-184). ops/ oms. https://www.who.int/mental_health/paho_guia_practicade_salud_mental.pdf

Rosa, J. D. (2012). Contesting representations of immigration. Anthropology News, 53(8), 13-14. http://linguisticanthropology.org/wp-content/uploads/2015/02/Jonathan-Rosa-Contesting-Representations-of-Immigration.pdf

Ruiz, J. M., Hamann, H. A., Mehl, M. R. \& O’Connor, M.-F. (2016). The Hispanic health paradox: from epidemiological phenomenon to contribution opportunities for psychological science. Group Processes E Intergroup Relations, 19(4), 462476. https://doi.org/10.1177/1368430216638540 
Sar, R. A. (2016). La construcción mediática de los inmigrantes en Iberoamérica. Revista Internacional de Comunicación y Desarrollo (RICD), 1(3), 25-39. http://dx.doi. org/10.15304/ricd.1.3.2946

Seminario, B., Palomino, L. \& Pastor, G. (2020). Perú: estimando el impacto macroeconómico de COVID-19. Foco Económico. http://focoeconomico.org/2020/05/08/peru-estimando-el-impacto-macroeconomico-de-covid-19/3

Serrano, M. \& Ibáñez, M. (2015). La prevención de las situaciones de impacto emocional en el ambiente laboral. Estudio teórico y análisis de su incidencia en el personal sanitario de la comunidad autónoma de Euskadi冈España. Trabajo y Sociedad, (25), 463-492. https://dialnet.unirioja.es/servlet/articulo?codigo $=5207411$

Stanley, N., Ellis, J., Farrelly, N., Hollinghurst, S., Bailey, S. \& Downe, S. (2017). "What matters to someone who matters to me": using media campaigns with young people to prevent interpersonal violence and abuse. Health Expectations, 20(4), 648-654. https://doi.org/10.1111/hex.12495

Surveillances, V. (2020). The epidemiological characteristics of an outbreak of 2019 novel coronavirus diseases (COVID-19)-China, 2020. CCDC Weekly, 2(8), 113-122. https://doi.org/10.46234/ccdcw2020.032

Torres López, T. M., López López, J. L., Mercado Ramírez, M. A. \& Tapia Curiel, A. (2014). Vivencias de migrantes mexicanos sobre estados emocionales experimentados durante su proceso migratorio y el consumo de alcohol y drogas. Estudios Fronterizos, 15(29), 247-270. https://doi.org/10.21670/ref.2014.29.a08

Valenzuela-Vergara, E. M. (2019). Media representations of immigration in the Chilean press: to a different narrative of immigration? Journal of Communication Inquiry, 43(2), 129-151. https://doi.org/10.1177/0196859918799099

Van Dijk, T. A. (2008). Escribir y hablar la inmigración. En M. Martínez (Ed.), Inmigración, discurso y medios de comunicación (pp. 13-14). Instituto Alicantino de Cultura Juan Gil-Albert. http:/ / rua.ua.es/dspace/handle/10045/16236

Vargas, G. (2019, 26 de noviembre). La tragedia de ser migrante y mujer: el caso de las mujeres venezolanas en Perú. Idehpucp. https://idehpucp.pucp.edu.pe/notas-informativas/la-tragedia-de-ser-migrante-y-mujer-el-caso-de-las-mujeres-venezolanas-en-peru/

Vargas Murga, H. (2014). Tipo de familia y ansiedad y depresión. Revista Médica Herediana, 25(2), 57-59. http://www.scielo.org.pe/scielo.php?script=sci_arttext\&pi$\mathrm{d}=\mathrm{S} 1018-130 \mathrm{X} 2014000200001$

Vázquez-Aguado, O. (1999). Negro sobre blanco: inmigrantes, estereotipos y medios de comunicación. Comunicar, (12), 55-60. https://doi.org/10.3916/C12-199909

Velarde Ramirez, M. A. (2019). Representación mediática de la dicotomía xenofobia-endofobia de la migración venezolana en el Perú del año 2017 al 2018 [Tesis de licenciatura, Universidad San Ignacio de Loyola]. http://repositorio.usil.edu.pe/handle/ USIL/9179

Venigalla, A. S. M., Vagavolu, D. \& Chimalakonda, S. (2020). Mood of India during COVID-19 - An interactive web portal based on emotion analysis of Twitter data. arXiv https://arxiv.org/abs/2005.02955 
Vidyadhara, S., Chakravarthy, A., Pramod Kumar, A., Sri Harsha, C. \& Rahul, R. (2020, 12 de mayo). Mental health status among the South Indian pharmacy students during COVID-19 pandemic quarantine period: a cross-sectional study. medRxiv. https://doi. org/10.1101/2020.05.08.20093708

Wang, W. (2006). Chinese governmental post-crisis management of 2003 SARS epidemic: evaluation of governmental communication strategies and frame correlation between government and mass media [Tesis de maestría, Virginia Polytechnic Institute and State University]. https://vtechworks.lib.vt.edu/bitstream/handle/10919/42731/ WeiruiThesis.pdf?sequence $=1$

Wenham, C., Smith, J. \& Morgan, R. (2020). COVID-19: the gendered impacts of the outbreak. The Lancet, 395(10227), 846-848. https://doi.org/10.1016/S01406736(20)30526-2

Women's link worldwide, Amnistía Internacional \& International Planned Parenthood Federation. (2020). Guía para proteger los derechos de mujeres y niñas durante la pandemia de COVID-19. Women's link worldwide. https://www.womenslinkworldwide.org/files/3112/guia-para-proteger-los-derechos-de-mujeres-y-ninasdurante-la-pandemia-de-covid-19.pdf

World Health Organization (wHO). (2020). Infection prevention and control during health care when novel coronavirus $(n \mathrm{CoV})$ infection is suspected. Interim guidance 19 March 2020. https://www.who.int/publications/i/item/infection-prevention-and-control-during-health-care-when-novel-coronavirus-(ncov)-infection-is-suspected-20200125

Yang, H. \& Ma, J. (2020). How an epidemic outbreak impacts happiness: factors that worsen (vs. protect) emotional well-being during the Coronavirus Pandemic. Psychiatry Research, 289, artículo 113045. https://doi.org/10.1016/j.psychres.2020.113045

Miguel Angulo-Giraldo

Peruano. Magíster en estudios latinoamericanos por la Universidad Federal de Integración Latinoamericana (Brasil). Investigador asociado y responsable de investigación de ciencias humanas de la Universidad Científica del Sur, Lima, Perú. Líneas de investigación: medios de comunicación, interculturalidad y espacios digitales. Publicación reciente: Angulo-Giraldo, M. \& Bolo-Varela, O. (2021). Medios de comunicación y conflictos sociales durante la pandemia por COVID-19: análisis de los enfoques presentes en la prensa de Lima durante las protestas contra el gobierno interino de Manuel Merino (2020). Desde el Sur, 13(1), e0005-e0005. https://doi. org/10.21142/DES-1301-2021-0005 
Luis Guanipa-Ramírez

Venezolano. Magíster en docencia para educación superior por la Universidad Nacional Experimental Rafel María Baralt (Venezuela) y licenciado en filosofía por la Universidad Católica Cecilio Acosta (Venezuela). Docente de cursos de investigación de la Universidad Científica del Sur, Lima, Perú. Líneas de investigación: educación, recursos humanos, filosofía, pensamiento complejo. Publicación reciente: Guanipa Ramírez, L. F. \& Angulo Giraldo, M. A. (2020). La identidad social en la educación: hacia una participación ciudadana, Desde el Sur, 12(1). http://dx.doi.org/10.21142/ des-1201-2020-0010

Jose Albites-Sanabria

Peruano. Magíster en ingeniería biomédica de la Universidad de Brown (EuA) e ingeniero mecatrónico de la Universidad Nacional de Ingeniería (Perú). Investigador asociado y responsable de investigación de ciencias empresariales de la Universidad Científica del Sur, Lima, Perú. Líneas de investigación: ingeniería, tecnología y biomedicina. Publicación reciente: Colina Ysea, F. \& Albites Sanabria, J. L. (2020). Aprendizaje e innovación: retos en las organizaciones del siglo xxi. Desde el Sur, 12(1). http://dx.doi.org/10.21142/des-1201-2020-0011 